\title{
Ab-initio investigation of structure and cohesive energy of crystalline urea
}

\author{
B. Civalleri ${ }^{1, *}$, K. Doll ${ }^{2}$ and C. M. Zicovich-Wilson ${ }^{3}$ \\ ${ }^{1}$ Dipartimento di Chimica IFM and NIS Centre of Excellence, Università di Torino, Via P. Giuria 7, \\ 10125 Torino, Italy \\ ${ }^{2}$ Institut für Mathematische Physik, Technische Universität Braunschweig, Mendelssohnstrasse, 3, \\ 38106 Braunschweig, Germany \\ 3 Facultad de Ciencias, Universidad Autonoma del Estado de Morelos, Av. Universidad 1001, \\ 62210 Cuernavaca, Mexico
}

\section{Supplementary material}

Table S1 Lattice parameters (in $\AA$ ) and atomic fractional coordinates of crystalline urea computed with different Hamiltonians. Basis set: $6-31 G(d, p)$. C and $\mathrm{O}$ atoms are on positions $(0,1 / 2, z), N$ and $\mathrm{H}$ atoms are on positions $(\mathrm{x}, \mathrm{x}+1 / 2, \mathrm{z})$. Space group: $\mathrm{P}-42_{1} \mathrm{~m}$.

\begin{tabular}{cccccccc}
\hline & HF & SVWN & PW91 & PBE & PBE0 & B3LYP & Exp. $^{\text {a }}$ \\
\hline$a$ & 5.949 & 5.257 & 5.598 & 5.604 & 5.588 & 5.675 & 5.565 \\
$c$ & 4.750 & 4.521 & 4.659 & 4.669 & 4.649 & 4.682 & 4.684 \\
& & & & & & & \\
$\mathrm{C}_{z}$ & 0.3281 & 0.3145 & 0.3282 & 0.3276 & 0.3272 & 0.3287 & 0.3260 \\
$\mathrm{O}_{z}$ & 0.5886 & 0.5994 & 0.6035 & 0.6023 & 0.5997 & 0.6001 & 0.5953 \\
$\mathrm{~N}_{x}$ & 0.1353 & 0.1547 & 0.1460 & 0.1460 & 0.1450 & 0.1435 & 0.1459 \\
$\mathrm{~N}_{z}$ & 0.1802 & 0.1644 & 0.1771 & 0.1768 & 0.1769 & 0.1784 & 0.1766 \\
$\mathrm{H} 1_{x}$ & 0.2386 & 0.2765 & 0.2595 & 0.2594 & 0.2575 & 0.2543 & 0.2575 \\
$\mathrm{H} 1_{z}$ & 0.2820 & 0.2768 & 0.2821 & 0.2820 & 0.2819 & 0.2824 & 0.2827 \\
$\mathrm{H} 2_{x}$ & 0.1339 & 0.1521 & 0.1433 & 0.1436 & 0.1428 & 0.1412 & 0.1441 \\
$\mathrm{H} 2_{z}$ & -0.0295 & -0.0640 & -0.0423 & -0.0424 & -0.0410 & -0.0383 & -0.0380 \\
\hline
\end{tabular}

${ }^{a}$ Swaminathan, S.; Craven, B. N.; McMullan, R. K. Acta Crystallogr. Sec. B 1984, 40, 300. 
Table S2 Lattice parameters (in $\AA$ ) and atomic fractional coordinates of crystalline urea computed at the B3LYP level of theory with different basis sets. $\mathrm{C}$ and $\mathrm{O}$ atoms are on positions $(0,1 / 2, \mathrm{z}), \mathrm{N}$ and $\mathrm{H}$ atoms are on positions $(\mathrm{x}, \mathrm{x}+1 / 2, \mathrm{z})$. Space group: $\mathrm{P}-42{ }_{1} \mathrm{~m}$.

\begin{tabular}{cccccc}
\hline & $6-31 \mathrm{G}(\mathrm{d}, \mathrm{p})$ & $\mathrm{DZP}$ & $6-311 \mathrm{G}(\mathrm{d}, \mathrm{p})$ & TZP & Exp. $^{\mathrm{a}}$ \\
\hline$a$ & 5.675 & 5.700 & 5.743 & 5.841 & 5.565 \\
$c$ & 4.682 & 4.678 & 4.707 & 4.710 & 4.684 \\
$\mathrm{C}_{z}$ & 0.3287 & 0.3303 & 0.3298 & 0.3372 & 0.3260 \\
$\mathrm{O}_{z}$ & 0.6001 & 0.6025 & 0.5982 & 0.6013 & 0.5953 \\
$\mathrm{~N}_{x}$ & 0.1435 & 0.1431 & 0.1415 & 0.1390 & 0.1459 \\
$\mathrm{~N}_{z}$ & 0.1784 & 0.1796 & 0.1798 & 0.1834 & 0.1766 \\
$\mathrm{H} 1_{x}$ & 0.2543 & 0.2544 & 0.2510 & 0.2467 & 0.2575 \\
$\mathrm{H} 1_{z}$ & 0.2824 & 0.2844 & 0.2826 & 0.2854 & 0.2827 \\
$\mathrm{H} 2_{x}$ & 0.1412 & 0.1398 & 0.1393 & 0.1368 & 0.1441 \\
$\mathrm{H} 2_{z}$ & -0.0383 & -0.0390 & -0.0355 & -0.0315 & -0.0380 \\
\hline
\end{tabular}

${ }^{a}$ Swaminathan, S.; Craven, B. N.; McMullan, R. K. Acta Crystallogr. Sec. B 1984, 40, 300. 
Table S3 CPU time ${ }^{a}$ (in sec) for SCF and gradients calculation on the first optimization step and total CPU time of the geometry optimization. The experimental structure $^{\mathrm{b}}$ was used as starting geometry. Space group: $\mathrm{P}-42{ }_{1} \mathrm{~m}$.

\begin{tabular}{lccc}
\hline & $\mathrm{T}_{\mathrm{CPU}} \mathrm{SCF}^{\mathrm{c}}$ & $\mathrm{T}_{\mathrm{CPU}}$ Gradients & $\mathrm{T}_{\mathrm{CPU}}$ Geom. Opt. \\
\hline Basis set: 6-31G(d,p) & $376(19)$ & 132 & $3570(11)$ \\
HF & $80(18)$ & 58 & $1011(11)$ \\
SVWN & $97(17)$ & 61 & $1308(13)$ \\
PW91 & $97(17)$ & 61 & $1302(13)$ \\
PBE & $326(16)$ & 162 & $3849(13)$ \\
PBE0 & $327(16)$ & 161 & $3928(13)$ \\
B3LYP & & & \\
& & & $3928(13)$ \\
Hamiltonian: B3LYP & $327(16)$ & 161 & $15869(14)$ \\
6-31G(d,p) & $1493(16)$ & 393 & $13266(15)$ \\
DZP & $1202(16)$ & 453 & $54237(15)$ \\
6-311G(d,p) & $4557(15)$ & 1367 & \\
TZP & & & \\
\hline
\end{tabular}

${ }^{\text {a }}$ Calculations carried out on 8 processors Itanium-2 1.3GHz SGI-Altix 350.

${ }^{\mathrm{b}}$ Swaminathan, S.; Craven, B. N.; McMullan, R. K. Acta Crystallogr. Sec. B 1984, 40, 300.

${ }^{\mathrm{c}}$ Number of cycles in parentheses

${ }^{\mathrm{d}}$ Number of geometry optimization steps in parentheses 